\title{
Scanning Probe Microscopy: Delineating Structure-Property-Processing Relationships in Hybrid Organic/Inorganic Nanomaterials
}

\author{
Nathan L. Yoder and Mark C. Hersam \\ Materials Science and Engineering, Northwestern University \\ 2220 Campus Drive, Evanston, IL 60208-3108 USA \\ WWW: http://www.hersam-group.northwestern.edu/
}

E-mail: n-yoder@northwestern.edu

The Hersam Research Group develops scanning probe microscopy nanofabrication and nanocharacterization techniques for hybrid organic/inorganic materials and devices. Ongoing research topics include silicon-based molecular electronics [1-5], organic light emitting diodes [6,7], molecular rotors [8,9], nanopatterned sensors [10,11], encapsulated carbon nanotubes [12,13], and catalytic oxides [14]. In all cases, the interplay between the organic and the inorganic subcomponents influences the overall structure and properties of the hybrid nanomaterial. Consequently, nanoscale characterization of organic/inorganic interfaces is required to develop structure-property relationships in these systems. Furthermore, nanometer scale processing techniques enable optimization of the performance of hybrid organic/inorganic devices.

As a case study of our research approach to nanomaterials science and engineering, this talk will focus on the application of the structure-property-processing paradigm to silicon-based molecular electronic materials and devices. A homebuilt ultra-high vacuum (UHV) scanning tunneling microscope (STM) allows individual molecules to be imaged, addressed, and manipulated on semiconducting surfaces with atomic resolution at temperatures from $8 \mathrm{~K}$ to $300 \mathrm{~K}$ [15]. This study builds on our previous research, in which spectroscopic characterization of individual molecules on degenerate $\mathrm{Si}(100)$ surfaces (both $\mathrm{n}$ - and $\mathrm{p}$-type) showed negative differential resistance (NDR) events. However, the overall reliability of these molecular junctions is of critical importance to potential devices, and consequently warrants further investigation. The stability of different classes of organic molecules is studied, including cyclopentene, styrene, 4-methoxy-styrene, and 5-vinyl-2,3-dihydrobenzofuran. Different modes of instability were observed, ranging from pure lateral motion to complete desorption from the surface. In the case of 4-methoxy-styrene, tuning the intramolecular degrees of freedom effectively eliminated the lateral motion entirely under the conditions studied. In the case of cyclopentene, the molecule desorbs cleanly from the surface, and the desorption yield has been measured as a function of sample bias and current. The desorption rate scales roughly linearly with applied sample bias, which indicates a single-electron process. Density 
functional theory (DFT) calculations have been performed to gain further insight into the electron and nuclear dynamics.

\section{References}

[1] N. P. Guisinger, et al, Proc. Nat. Acad. Sci. USA, 102, 8838 (2005).

[2] R. Basu et al, Appl. Phys. Lett., 85, 2619 (2004).

[3] M. C. Hersam and R. G. Reifenberger, MRS Bull., 29, 385 (2004).

[4] N. P. Guisinger, et al, Nanotechnology, 15, S452 (2004).

[5] N. P. Guisinger, et al, Nano Lett., 4, 55 (2004).

[6] L. S. C. Pingree, et al, Appl. Phys. Lett., 86, 073509 (2005).

[7] L. S. C. Pingree, et al, Appl. Phys. Lett., 85, 344 (2004).

[8] R. Basu, et al, J. Vac. Sci. Technol. B, 23, 1785 (2005).

[9] B. Wang, et al, Nanotechnology, 15, 324 (2004).

[10] C. R. Kinser, et al, Nano Lett., 5, 91 (2005).

[11] M. E. Greene, et al, Microscopy Research and Technique, 64, 415 (2004).

[12] M. S. Arnold, et al, Nano Lett., 5, 713 (2005).

[13] M. S. Arnold, et al, Nano Lett., 3, 1549 (2003).

[14] M. E. Greene, et al, Adv. Mater., 17, 1765 (2005).

[15] E. T. Foley, et al, Rev. Sci. Instrum., 75, 5280 (2004).

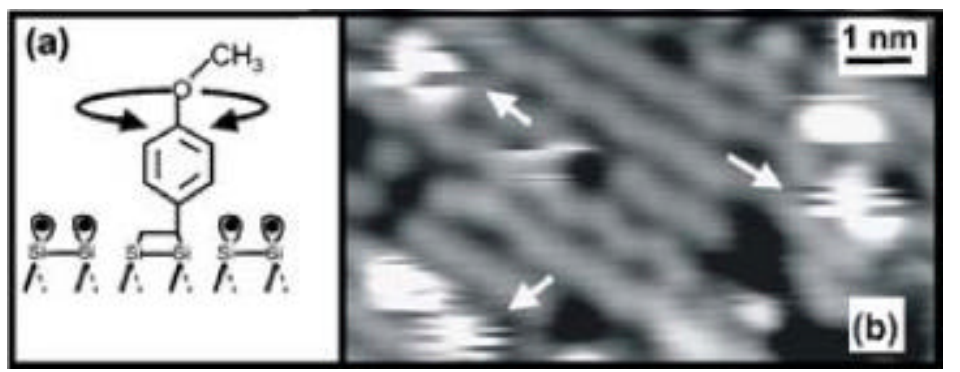

Figure 1: (a) Schematic of $(2+2)$ cycloaddition of a 4-methoxystyrene molecule on a dimer on the clean $\mathrm{Si}(100)$ surface [8]. (b) UHV STM image of 4-methoxystyrene molecules on the clean $\mathrm{Si}(100)$ surface following a dose of $0.7 \mathrm{~L}(-2.0 \mathrm{~V}, 0.1 \mathrm{nA})$. Arrows indicate multiple switching events.

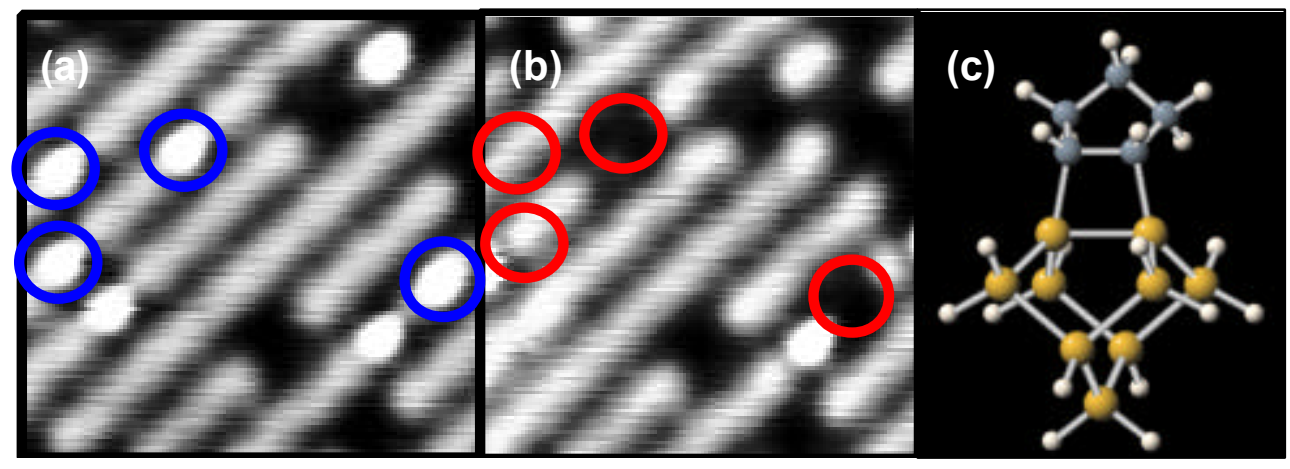

Figure 2: STM Images of cyclopentene bound to a clean silicon surface $(-2 \mathrm{~V}, 0.1 \mathrm{nA})$ (a) before desorption (b) after scanning at $-4 \mathrm{~V}, 2 \mathrm{nA}$ with desorbed molecules circled (c) Model of cyclopentene bonded to a small silicon cluster for the purposes of the DFT calculations. 